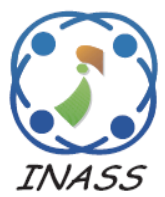

\title{
A Novel FDCT-SVD Based Watermarking with Radon Transform for Telemedicine Applications
}

\author{
Ahmed A Mohammed ${ }^{1 *}$ \\ Mohammed A M Abdullah ${ }^{1}$ \\ Faris S. Alghareb ${ }^{1}$ \\ Sohaib R. Awad ${ }^{1}$ \\ ${ }^{I}$ Ninevah University, Iraq \\ * Corresponding author’s Email: ahmed.mohammed@uoninevah.edu.iq
}

\begin{abstract}
Digital Watermarking has been widely employed for content authentication by securely embedding patient private information within medical images. In this work, we present a novel watermarking, which employs hybrid Multiscale/Multiresolution frequency coefficients selection using the Fast Discrete Curvelet Transform (FDCT) in conjunction with Singular Value Decomposition (SVD). In order to add an extra layer of security, the Radon Transform (RT) is applied on the watermarks before embedding for the sake of robustness and security due to RT properties of capturing image directionality which is essential against various attacks. The proposed method attained promising results and has shown that the imperceptibility of watermarked medical images is higher than $55 \mathrm{~dB}$ for all medical images. In addition, the scheme has shown an improved robustness compared with existing related work.
\end{abstract}

Keywords: Digital watermarking, Fast discrete curvelet transform, Radon transform, Medical images, Data hiding, Imperceptibility.

\section{Introduction}

Digital watermarking is crucial in telemedicine and medical image protection especially during the covid-19 pandemic. Telemedicine can be defined as the process of exchanging medical images and information amongst medical organizations to enable global access to limited medical experts. It is worth pointing out that the integrity of medical information should always be protected during this exchange process. Digital watermarking has been widely employed for content authentication by securely embedding patient private information within medical images. In essence watermarking schemes are evaluated using two main categories, i.e., robustness and imperceptibility. Moreover, the performance of the schemes in terms of security should also be meticulously investigated. In this work we are concerned with digital media and our focus is on digital imaging, specifically medical images which are important in tracking the progress of an ongoing illness and patient follow-up [1]. Security issues are at rampage, as a consequence, healthcare organizations are looking to implement stringent policies to ensure data security when transferring patient's medical information from doctor to another. When an image is altered during transmission over an open-access network, this could deviate the diagnosis and create serious health issues for patients. Hence, it is imperative to ensure the integrity of medical images [2]. The integrity of medical images can be compromised by several security issues. For example, manipulating the medical image source or changing its legitimate owner is one of the security concerns; another problem arises when the patient's record is detached from its corresponding image. A promising solution would be the use of digital watermarking techniques with medical images to address the aforementioned issues [3]. In principle, two watermarking domains can be used namely, spatial and frequency domain. In the spatial domain, bits are added directly to the pixel values of the cover image by changing the pixel values of the image [4]. This technique has less complexity and is very easy to 
implement but the information inserted in pixel value can easily be detected by unauthorized persons. In addition, spatial domain watermarking is prone to attacks. On the other hand, in the transform frequency domain watermarking, the watermark is embedded in frequency/transform coefficients rather than the image pixels which makes it more robust compared to the spatial domain technique. However, these techniques are more complex than spatial domain [5]. There are several methods to perform the watermarking in the frequency domain such as discrete cosine transform (DCT), DWT, and discrete fourier transform (DFT), also other transform domains like singular value decomposition (SVD), hough, and the radon transform (RT), can be implemented to perform the watermarking [6]. The main issue with present medical images digital watermarking techniques is the trade-off between robustness, image perceptual quality, and security. Researchers mostly tend to keep a certain level of robustness to keep the imperceptibility and retaining a PSNR value of at least $45 \mathrm{~dB}$. Keeping that state of balance between these important three factors among other factors is crucial for a watermarking scheme to be reliable for medical images. [5]. This paper aims to design a robust yet imperceptible watermarking algorithm specially tailored for medical imaging. The main contributions of this work are: (1) a marriage between FDCT-SVD and radon transform (RT) is proposed to achieve high perceptibility while being robust to attacks; (2) two watermarks are embedded withing different frequency sub-bands for high reliability extraction; (3) radon transform (RT) is employed to increase the robustness of the watermarking scheme against geometrics attacks due to RT properties of capturing the directional features of the image; (4) the scheme is highly imperceptible as it averaged at PSNR of $57.61 \mathrm{~dB}$ which outperforms all the related work. The scheme has a significant advantage over other state-of-the-artschemes in terms of robustness as the proposed scheme provides supreme robustness, especially against cropping and random cropping attacks which is not utilized in the state-of-the-art work as the average value after such attacks is always above 0.9 . The paper organization is as follows: section 1 provided an overview of digital watermarking and embedding domains along with current challenges in the field medical images' watermarking. section 2 dives into the related works, state-of-the-art work, and the current drawbacks, this section also covered the difference between our scheme and state-of-theart work. while in section 3 the FDCT background is tackled, also our proposed scheme is defined in this section. In section 4 the experimental result, evaluations, and comparisons are laid out. Finally, section 5 sums up and concludes this paper.

\section{Literature review}

The are several watermarking methods proposed in the literature for example, JING LIU et al. [7] introduced a zero-watermarking scheme based on 3D dual-tree complex wavelet and 3D hyper-chaos, the scheme proposed based on the features selected from medical volume data. The scheme enhanced the NC value average by $46.67 \%$ against geometric attacks and fulfilled transmission and storage security of medical data but lacks imperceptibility recommended for medical images. Thakkar et al. [8] proposed a blind watermarking algorithm in the DWT-SVD domain, the scheme is intended for medical images security. The logo watermark and electronic patient record (EPR) are embedded into the singular values of DWT sub-bands. The scheme is designed to resist the most common attacks. This scheme has a limitation for being suitable only within the region of interest (ROI) of medical images. Parah et al. [9] presented a blind scheme that embeds EPR plus a watermark image, the latter is embedded within selected DCT coefficients of the host medical image, the error-correcting code (ECC) was conducted to the $E P R$ at the embedding process to diminish errors in the recovery process. The scheme has a significant drawback in terms of perceptual quality where the average PSNR is $41 \mathrm{~dB}$, which is barely acceptable for medical images watermarking. Nagpal et al. [10] proposed a watermarking scheme based on DWT, neural network, and rivest-shamir-adleman (RSA) encryption. In this technique, the watermark image was scrambled by applying the RSA encryption for security reasons. Further, the scrambled watermark is embedded into the DWT sub-bands of the host image by employing the artificial neural network (ANN) technique, the scheme performance is just acceptable. Kishore et al. [11] introduced a review study of several watermarking schemes that deal with medical images and also, presented a comparison among three watermarking schemes for medical images such as DWT-RSA, DWT-SVD, and DWT-ANN. The comparison indicates that the DWT-SVD scheme achieved better results, still the authors did not involve neither encryption based-techniques nor watermark image transformation-based technique in the comparison. Mahmood [12] proposed a nonreversible adaptive watermarking scheme that focuses on for medical images integrity. In this scheme, the host image was divided into several blocks, and by the use of segmentation map technique the region of background (ROB) is determined. Later, 
few low entropy blocks were selected for embedding after calculating the entropy of blocks. DCT is also performed on these blocks, the watermark image is embedded into the DCT coefficients by LSB technique. The limitation of this scheme is the low imperceptibility. Singh et al. [13] introduced two watermarking techniques based on cryptography, DWT, and SVD intended for medical images security. Three different ECCs were employed for encoding the payload. The three ECC are reed-solomon, bosechaudhuri-hocquenghem $(\mathrm{BCH})$, and hamming codes. Encoded payload is encoded into the DWT coefficients in the DWT-based scheme and SVDbased scheme, respectively, the scheme drawback is that it has a low capacity and the average PSNR value is $35 \mathrm{~dB}$ among different gain factors ranging from 0.01 to 1 . Abdullah et al. [14] introduced a novel watermarking scheme for protecting iris images, in order to protect the iris images, a binary EPR is inserted into the middle frequency band coefficients of the DCT of the host image, by interchanging multiple middle-frequency band coefficients pairs of the DCT randomly, the scheme is highly perceptual and robust but lacks the use of encryption/scrambling techniques with the embedded payolas. In [15] the authors proposed a watermarking scheme based on FDCT-DCT, block-wise DCT is performed for obtaining various frequency coefficients, the scheme is applied on different types of medical images. Result analysis illustrated that the perceptual quality of the watermarked images is acceptable as the PSNR value is above $45 \mathrm{~dB}$ for all medical images. Nevertheless, the scheme has some limitations with preserving high perceptual quality. The work in [16] proposed a reversible with a high-capacity watermarking scheme designed for the e-healthcare applications. The pixel to block (PTB) approach is employed in this work for being computational effective and an alternative to the interpolation of the host image, also, to improve a reversible scheme. The scheme embeds a fragile EPR payload with a block checksum ( $4 \times 4$ for each block) in the host image to detect any tampering and localize that tamper. The embedding domain is the intermediate significant bit substitution (ISBS) which is designed to overcome any usual LSB removal attack. The scheme is robust to some extent but in terms of perceptual quality the scheme achieved a PSNR value average of 46.36 for both medical and standard images and this can be a weakness point when it comes to medical images as the perceptual quality needs to be preserved extremely well. The authors in [17] proposed a watermarking scheme in DWT using SVD and particle swarm optimization (PSO) for the protection of medical images. An improved DWT (IDWT) is employed to the host image to obtain the invariant DWT. By using a threshold function, the watermark is embedded into the modified values of the DWT coefficients. PSO approach is used for scaling factors optimization. The latter scheme lacks the preserving of perceptual quality when compared to state-of-theart- works. Authors of [18] have proposed an adaptive technique for digital watermarking based on hybrid scheme (DCT-SVD) along with employing canny edge detector. The payload is encrypted and inserted into the edge-based vector which is obtained from the diagonal matrix of the DCT-SVD. The perceptual quality in terms of PSNR ranged from 51 to $55 \mathrm{~dB}$, also, robustness test results were very competitive. In [19] authors proposed a robust watermarking scheme based on FDCT, DCT, and SVD. FDCT is performed up to the 3rd level and three sub-bands obtained high, middle, and low frequency curvelet coefficients, the high frequency (HF) is selected. Later DCT-SVD is applied on HF, respectively. The DCT-SVD is performed on the payload too, the exchange between the host image singular value and watermark singular value is done. The scheme performed competitively in terms of imperceptibility and robustness. Authors of [20] a multipurpose watermarking scheme that serves for authentication, image recovery, and copyright protection, also the scheme utilizes the embedding of multi watermarks one for robustness purpose and the others for both authentication and restoration of the digital image. The robust watermark embedding uses the intermediate coefficients quotative property besides an adaptive selection for encoding strength in order to achieve balance between transparency and robustness. On the other hand, the fragile watermarks are inserted into the least significant bits based on an improved replacement technique. Though the technique has a PSNA average 41.8, but robustness wise, the scheme performed competitively. In [21] authors have proposed a two-transform based watermarking technique, namely discrete tchebichef and SVD transforms. For watermark security purposes the Arnold's transform is performed on the cover image, moreover, the cover image is transformed into the discrete tchebichef transform after being divided into small blocks. In order to decompose with the SVD, lowest discrete tchebichef transform coefficient from all blocks are collected. Finally, the payload is inserted into the singular SVD matrix. Result of the proposed scheme were competitive, where the average PSNR is $45.3 \mathrm{~dB}$ which still need more improvement, the scheme is very robust against various attack and over performed many schemes in the field, the SSIM is almost perfect which averaged at 0.9984 . Authors of [22] presented 
an image watermarking technique based on lifting wavelet transform with the SVD transform by which the intactness of embedded watermark is increased. The SVD is applied on the triangular matrix which is obtained by applying the schur transform on the low frequency subband of the wavelet. The scheme is robust and imperceptible, the main drawback is designated to resist non-geometrical attacks and never tested against geometrical attacks like rotation or cropping. In our previous work [5] we proposed a robust and secure scheme based on DCT-SVD where chaotic encryption is used for the security of the payload. The scheme was designed especially for medical images and the results were competitive in terms of medical image perceptual quality. Based on the previous works, the main drawback is the inability to achieve an adequate balance between preserving the high perceptual quality of the medical image (i.e. achieving an average PSNR of $55 \mathrm{~dB}$ or above) while maintaining reliable robustness against attacks. Our scheme differs from the aforementioned work by being highly imperceptible secure, yet robust. In this paper, we, therefore, address the previous drawbacks by proposing a new robust watermarking scheme in which we keep the original perceptual quality of the image. Although the scheme is designated for medical images, it can handle all other types of images with the same efficiency.

In this paper, we, therefore, address the previous drawbacks by proposing a new robust watermarking scheme in which we keep the original perceptual quality of the image.

\section{Proposed scheme}

FDCT is a multi-scale geometric transform, that can represent edges and curves singularities much more efficiently than traditional wavelets due to the wavelet's poor directionality. In 1999 an anisotropic transform named ridgelet transform was introduced by [23], later in 2000, a first generation curvelet transform was also introduced by the same authors. The 2nd generation has shown promising results in many image processing applications. The FDCT is very efficient in representing curve-like edges. The FDCT is implemented using two main ways, the unequally spaced fast fourier transform USFFT and wrapping function-based FDCT. In general, the FDCT is defined in Eq. (1) as:

$$
C(j, \theta, k 1, k 2)=\sum_{\substack{0 \leq x<M \\ 0 \leq y<N}} f[x, y] . \varphi_{j, \theta, k 1, k 2}[x, y]
$$

Where $j$ is the scale, $\theta$ is the orientation, $k 1, k 2$ are spatial locations of curvelets. $\varphi[\mathrm{x}, \mathrm{y}]$ is the curvelet

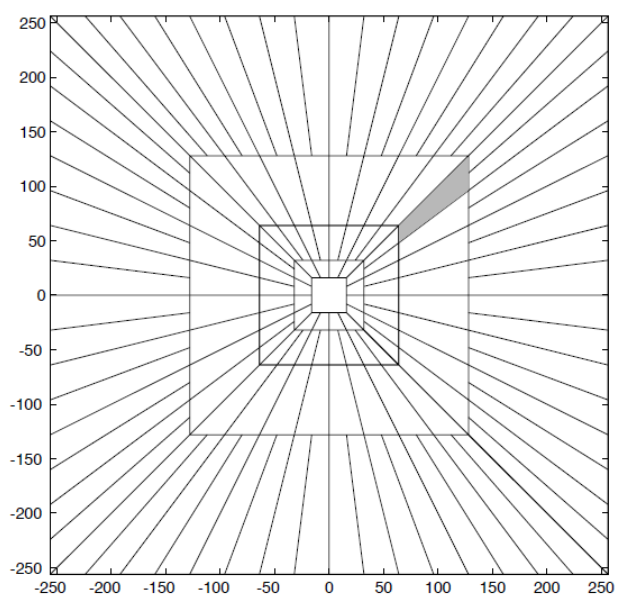

Figure. 1 Basic digital tiling
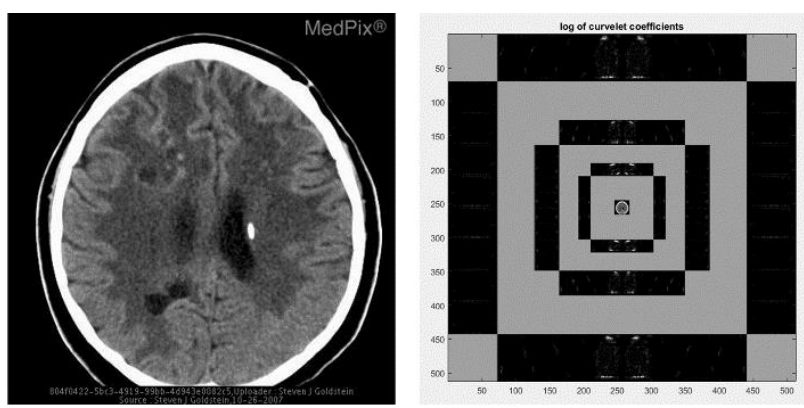

Figure. 2 Brain CT and its FDCT coefficients

basis function and $f[x, y]$ is the input image with $M X N$ dimensions [24], the curvelets in the pixel domain are organized in numerous orientations $\theta$ and scales $j$ so that the total FFT Plane of the image is enveloped to evade all kinds of signal loss as illustrated in Fig. 1 which shows a 5-level curvelet of an image. The wedge-shaped represents the FFT of the curvelet at the scale $j=4(C\{1,4\}\{1,4\})$ and the orientation of $\theta=5$. The approximation coefficients at the scale of $j=1(C\{1,1\}\{1,1\})$ is at the center square, while other wedges-shaped give detailed coefficients at different scales $j=2,3,4 \ldots .(C\{1, i\}\{1, i\})$. Practically, Fig. 2 illustrates a brain CT image and its DFCT coefficients.

In this work, a new semi-blind multiscale/multiresolution hybrid frequency coefficients selection-based watermarking scheme is proposed. The analysis of frequency wrapping based FDCT is performed on the host medical image while the decompositions are carried out up to the fourth level (scale 4 with 32 orientations) to get different resolutions, hybrid-band selection is adapted for multiscale/multiresolution frequency sub-bands selection (i.e. low-frequency curvelet coefficients $C\{1,1\}\{1,1\}$ from scale 1 and high-frequency curvelet coefficients $C\{1,1\}\{1, i\}$ from scale 4 where $i$ relates to different 32 orientations). This 
multiscale/multiresolution will serve later as alternatives for watermarks extraction as we are embedding two binary logo watermarks in the medical image. Finally, the SVD is applied for the selected curvelet frequency coefficients (high and low) to attain an embedding environment with high robustness, security and transparency due to the band multiresolution and the attractive mathematical properties of SVD, thus the scheme is employing hybrid frequency sub-bands. The SVD can be expressed in Eq. (2) as follows:

$$
A=U X S X V^{T}
$$

Where $U$ is the orthogonal matrix of the left singular vectors, $S$ is the diagonal matrix of singular values, and $V^{T}$ is the transpose matrix of $V$. The inverse SVD $A^{-1}$ can be obtained from Eq. (3) as:

$$
A^{-1}=V S^{-1} U^{T}
$$

On the other hand, the two watermarks were transformed into the radon transformation (RT) before embedding and inserted later in the selected multiscale/multiresolution sub-bands due to RT properties of capturing image directionality which is essential against various attacks as illustrated before. The RT of a two-dimensional image $f(x, y)$ can be defined in Eq. (4) as:

$$
\left.R f(t, \theta)=\iint f(x) \delta\left(\left\langle x, n_{0}\right\rangle\right)-t\right) d x
$$

Where $n_{0}=(\cos \theta, \sin \theta)$ which is the unit vector. And the inverse RT can be calculated from Eq. (5) as follows:

$$
f(x)=\frac{1}{2 \pi} \int_{0}^{\pi}(R f(., \theta) * h)\left(\left\langle x, n_{0}\right\rangle d \theta\right.
$$

where $h$ is such that $\hat{h}(\omega)=|\omega|$

After testing our method under various attacks, the proposed scheme showed very promising results compared to the other state-of-the-art works. The diagram of the proposed scheme is illustrated in Fig. 3.

\subsection{Embedding steps}

The scheme targets the multiscale/multiresolution properties of the FDCT by employing these properties watermark logos are inserted into those different frequency coefficients of the FDCT where we inserted two logo watermarks in the low frequency curvelets coefficients and the other one in

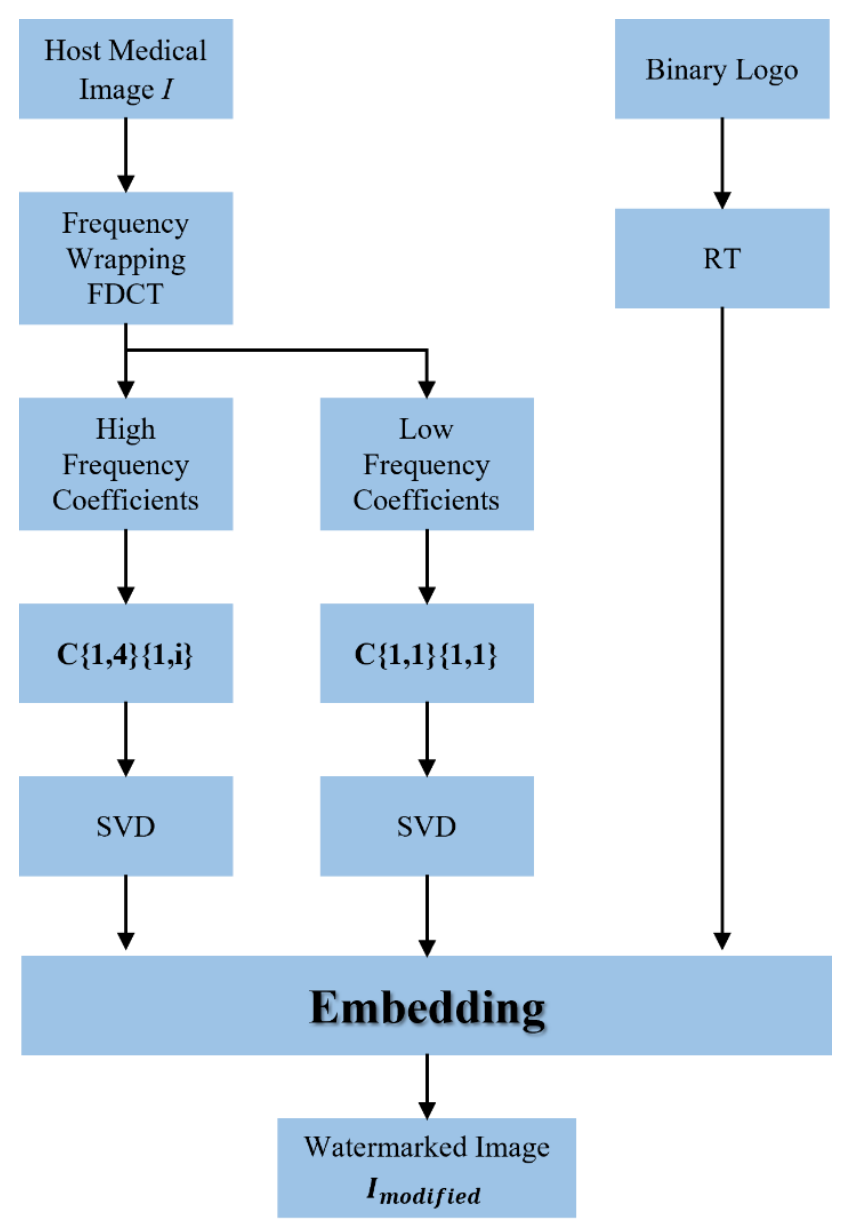

Figure. 3 The proposed watermarking scheme diagram

the high frequency curvelets coefficients. First, the binary logo denoted by $W$ is read which has a size of $64 \times 64$ and denote it by $W$, later $W$ is transformed into the RT. On the other hand, a $1024 \times 1024$ host image is read to apply the frequency wrapping based FDCT up to scale 4 . Both low and high-frequency curvelets frequency coefficients $C\{1,1\}\{1,1\}$ from scale 1 and $C\{1,1\}\{1, i\}$ from scale 4 respectively, are selected to perform SVD to prepare our embedding domain. The watermarks are inserted into the diagonal matrix of the SVD and later the inverse SVD is performed with the inverse FDCT to obtain the final watermarked image $I_{\text {modified }}$. The details are explained in detail in algorithm 1 .

\footnotetext{
Algorithm 1: Embedding Algorithm

Input: $I, W, \alpha$.

(I: host Image, $W$ : binary watermark logo, $\alpha$ : gain factor)

Output: $I_{\text {Modified }}$

( $I_{\text {modified }}:$ watermarked Image)

1. Read the binary logo watermark with the size of 64X64 and denote it by $W$

2. Transform $W$ into the RT in Eq. (4) to get the sinogram and denote it by $W_{R T}$
} 
3. Read host medical image 1024X1024 and denote it by $I$

4. Apply the frequency wrapping based FDCT in Eq. (1) up to scale 4.

5. Perform SVD in Eq. (2) on Low-frequency curvelet coefficients obtained from Eq. (1)

6. Embed the watermark in S1 diagonal matrix obtained from Eq. (2).

where $\alpha=0.2$

$$
S 1_{\text {modified }}=S 1+\alpha * W_{R T}
$$

7. Apply inverse SVD in Eq. (3) to get $S V D_{\text {modified (1) }}$

8. Perform SVD in Eq. (2) on High-frequency curvelet coefficients

9. Embed the second watermark in $S 2$ diagonal matrix obtained from Eq. (2). where $\alpha=0.9$.

$$
S 2_{\text {modified }}=S 2+\alpha * W_{R T}
$$

10. Apply inverse SVD Eq. (3) to get $S V D_{\text {modified(2) }}$

11. Apply inverse frequency wrapping based FDCT and get the final watermarked image and denote it by $I_{\text {modified } 2}$

\subsection{Extraction steps}

The extractions process is basically the inverse of the embedding process and these steps are explained in details in algorithm 2.

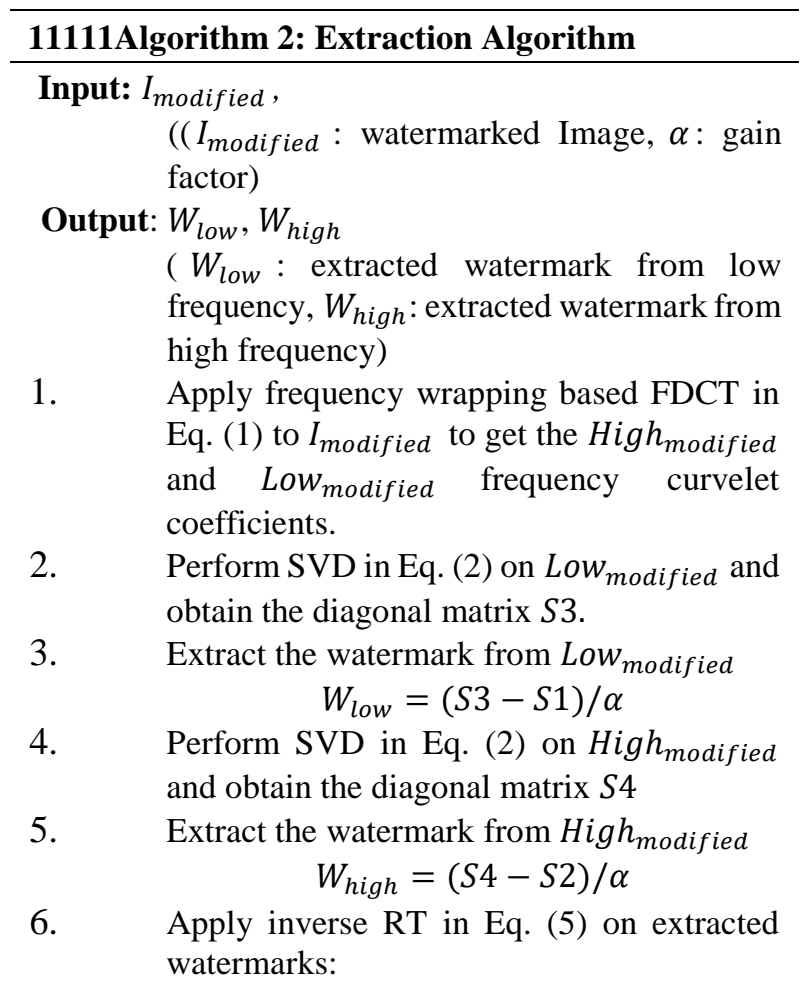

\section{Experimental results}

The work is conducted under a PC with Intel 2.5 $\mathrm{GHz}$ core $\mathrm{i} 7 \mathrm{cpu}$ with $4 \mathrm{~GB}$ of ram under the MATLAB environment. Experiments are conducted using the MedPix database [25] which is an openaccess database that contains nearly 59,000 images. We used four types of medical images (X-ray, MRI, CT scan, and ultrasound). The average execution time for the embedding process is 1.21 seconds, while the extraction process takes 1.54 seconds. To evaluate the proposed scheme, two metrics are used namely: PSNR and NC.

The imperceptibility in watermarking can be defined as how similar the original host image when compared to its the watermarked version. For evaluating this similarity, a parameter like a peak signal to- noise ratio (PSNR). To measure the

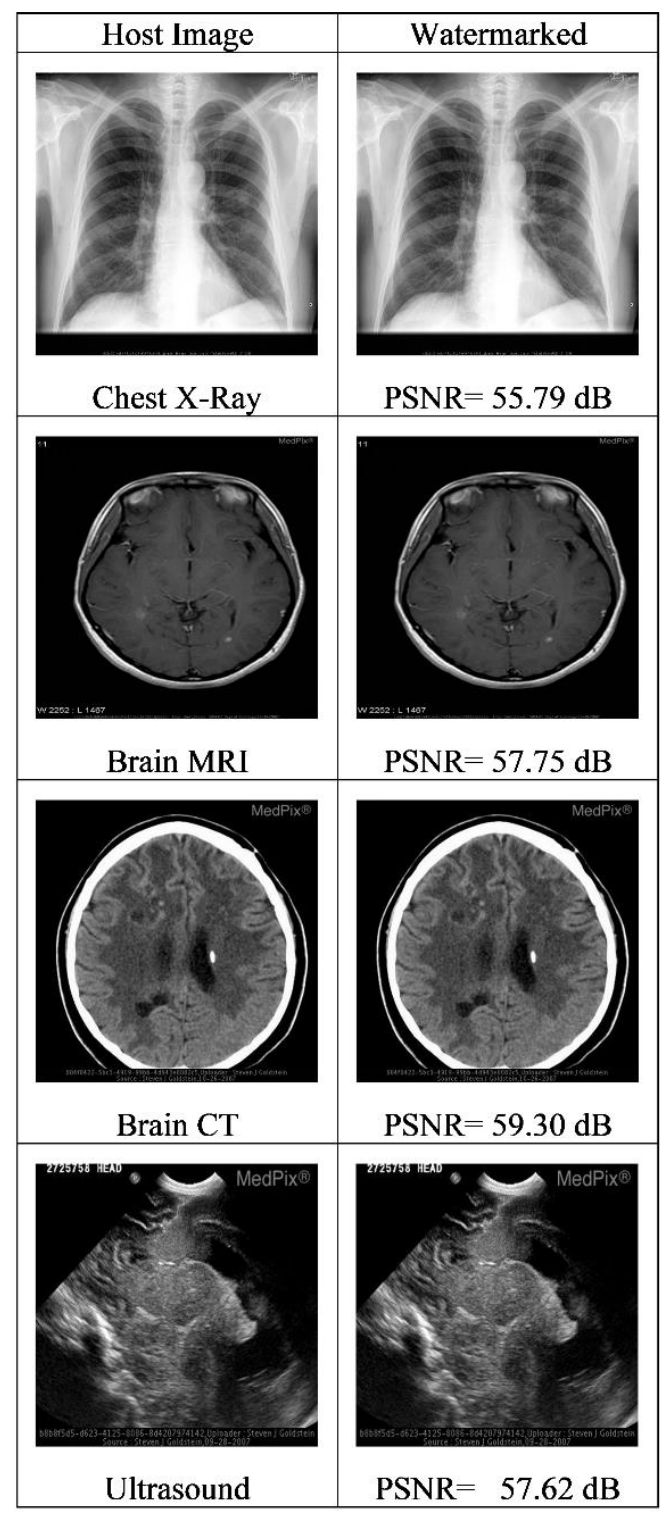

Figure. 4 Original medical images and their watermarked versions with their PSNR values 
similarity between the original and extracted watermark the $\mathrm{NC}$ comes in. If the $\mathrm{NC}$ value is equal to one, then both the images are the same. $\mathrm{NC}$ value lies between 0 and 1 .

\subsection{Scheme performance}

Fig. 4 presents the original and the host medical images with their corresponding watermarked images each with a PSNR value measured after embedding. It can be clearly seen from Fig. 4, the watermarking results in terms of imperceptibility are substantial, all PSNR values of watermarked medical images vary from $55 \mathrm{~dB}$ to $59 \mathrm{~dB}$. This perceptual quality is crucial especially for medical images as it guarantees no distortions or artifacts of any kind are presented after the watermarking process is performed. As for the watermark extraction process, two watermarks have been extracted as illustrated previously, and therefore, both of the extracted watermarks are denoted by $W_{\text {high }}$ and $W_{\text {low }}$ according to their embedding curvelet frequency coefficients (i.e., high and low), these extracted watermarks are not binary as they were before the embedding. This is because the inverse RT yields an image in double precision representation with some artifacts like some blurring. To compare them with the original embedded binary logo they need to be transformed into the binary form and enhanced for objective and subjective comparison. Fig. 5 illustrates the original and the extracted watermarks ( $W_{\text {high }}$ and $W_{\text {low }}$ ) with the introduced artifacts.

As depicted in Fig. 5, despite the use of a smooth theta for the sampling $(0: 1: 179)$ in the forward and inverse RT, the reconstructed watermarks $W_{\text {High }}$ and $W_{\text {Low }}$ using the inverse RT does not match the original embedded binary logo. For example, some obvious artifacts can be noticed due to the inverse RT with filtered backpropagation like the frame-shaped rectangle around the watermark, blurring effect, etc. To overcome this issue and approximate the originally embedded watermark, we use two main processing steps to convert the output to binary and remove the artifact window. The first step is to transform the reconstructed watermark image into the binary form, while the second step is to define a

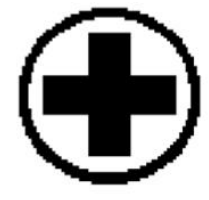

Original

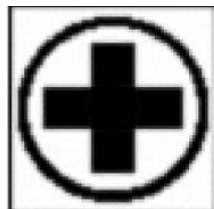

$W_{\text {high }}$

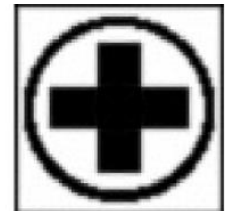

$W_{\text {low }}$
Figure. 5 The original and the extracted watermarks $\left(W_{\text {high }}\right.$ and $\left.W_{\text {low }}\right)$ with the introduced artifacts certain threshold for the binary image from step 1, the threshold value has been empirically set to 0.4 . The aforementioned steps are explained in the pseudo in algorithm 3. Fig. 6 shows the result after applying algorithm 3 . The watermark resulted from step 2 gave the best result with an NC value of 0.9995 and 0.9979 for $W_{\text {High }}$ and $W_{\text {Low }}$, respectively.

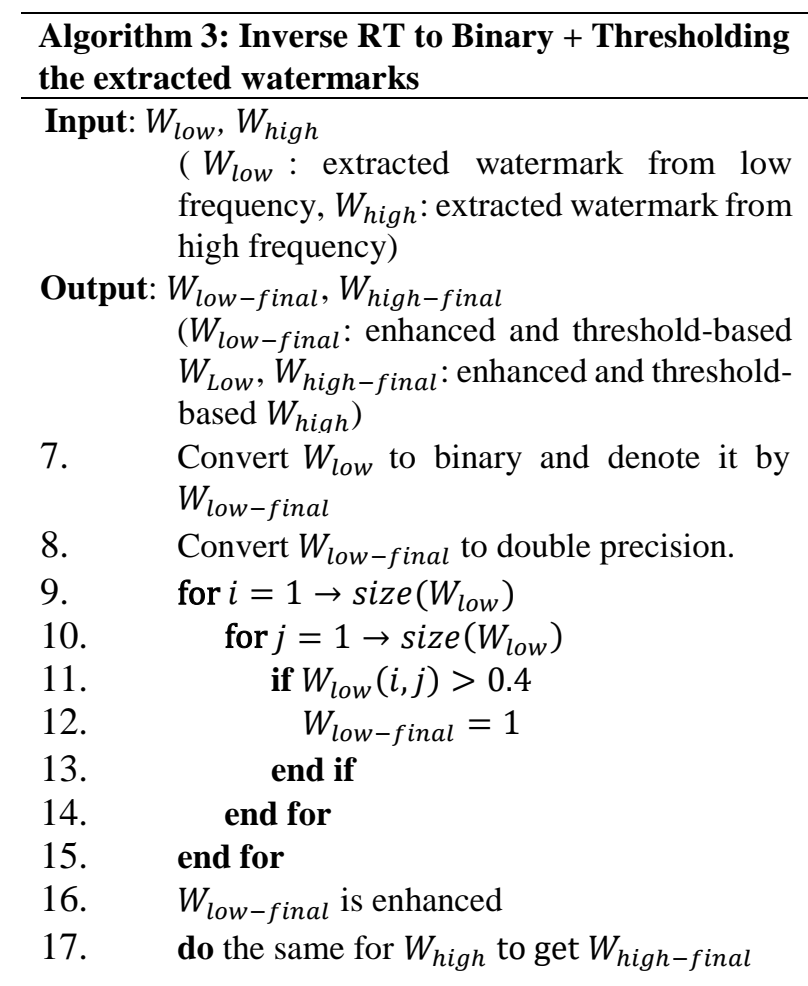

Fig. 7 shows the extracted watermarks for the $\mathrm{x}$-ray image against Gaussian noise attack with mean 0 and variance 0.1 , JPEG compression attack with quality factor of $1 \%$, two random cropping attacks, and rotation attack. The scheme performance is very promising and the NC values for selected watermarks are greater than 0.9 . The quality factor of compressed image with JPEG ranged from $90 \%$ to $25 \%$ and up to $1 \%$ which means a big loss in the image information and only reliable watermarking schemes can survive such attack. The watermark extractions along different quality factors were substantial as the NC value of the extracted watermark from low-frequency coefficients was above 0.9 under different quality

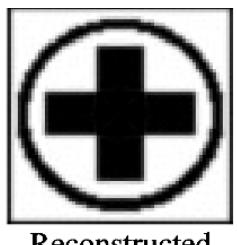

Reconstructed

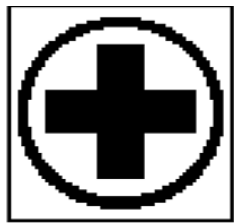

Binary form

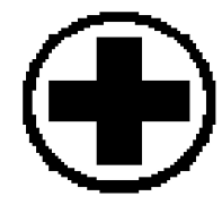

Threshold Based
Figure 6 Inverse RT to binary and thresholding the extracted watermarks 
Table 1. Robustness performance in NC values for all medical test images against various attacks

\begin{tabular}{|l|c|c|c|c|c|c|}
\hline \multirow{2}{*}{\multicolumn{1}{|c|}{ Attack }} & \multicolumn{2}{c|}{ Brain MRI } & \multicolumn{2}{c|}{ Brain CT } & \multicolumn{2}{c|}{ Ultrasound } \\
\cline { 2 - 7 } & High & Low & High & Low & High & Low \\
\hline No Attack & 0.9985 & 0.9969 & 0.9995 & 0.9938 & 0.9990 & 0.9933 \\
\hline Gaussian Noise, V=0.01 & 0.7276 & 0.9263 & 0.7537 & 0.9193 & 0.7414 & 0.9558 \\
\hline Gaussian Noise, V=0.1 & 0.7049 & 0.9090 & 0.7149 & 0.8207 & 0.7092 & 0.8815 \\
\hline Rotation 45 & 0.9851 & 0.8069 & 0.9969 & 0.7774 & 0.9974 & 0.8274 \\
\hline Histogram equalization & 0.8835 & 0.8486 & 0.8835 & 0.8486 & 0.8820 & 0.9312 \\
\hline JPEG, QF=90\% & 0.9959 & 0.9877 & 0.9938 & 0.9995 & 0.9928 & 0.9990 \\
\hline JPEG, QF=25\% & 0.9785 & 0.9902 & 0.9897 & 0.9985 & 0.9851 & 0.9990 \\
\hline JPEG, QF=1\%, & 0.9248 & 0.9568 & 0.8699 & 0.9872 & 0.9243 & 0.9877 \\
\hline Salt and Pepper Nosie, D=0.01 & 0.7049 & 0.9085 & 0.7243 & 0.8962 & 0.7134 & 0.9362 \\
\hline Median Filtering 2X2 & 0.9882 & 0.9846 & 0.9985 & 0.9831 & 0.9887 & 0.9995 \\
\hline Median Filtering 3X3 & 0.9923 & 0.9699 & 0.9995 & 0.9913 & 0.9897 & 0.9902 \\
\hline Median Filtering 9X9 & 0.9643 & 0.9110 & 0.9979 & 0.9263 & 0.9990 & 0.9303 \\
\hline
\end{tabular}

factors even in the worst-case scenario of $1 \%$ quality factor. Scheme performance against JPEG and histogram equalization attack is illustrated in Table 1. In addition, Table 1 illustrates the effects of more attacks on the scheme performance using other types of medical images. Results again are promising and an extraction alternative with an $\mathrm{NC}$ value of 0.9 is always present. Finally, the random crop attack is applied and the results are shown in Fig. 7, this attack seriously damages the image and it could hit anywhere in the image, we took this attack to the next level by cropping almost $90 \%$ of the image, the scheme performed magnificently and the embedded watermarks were extracted successfully where $90 \%$ crop is applied from inside out leaving only the borders of the image and vice versa. Results were perfect and the extracted watermark with an $\mathrm{NC}$ value

Table 2. Imperceptibility and robustness comparison with other works without attacks for medical images

\begin{tabular}{|c|c|c|c|}
\hline Scheme & Image & PSNR & NC \\
\hline & X-Ray & $\mathbf{5 5 . 7 9}$ & $\mathbf{0 . 9 9 9 5}$ \\
Proposed & MRI & $\mathbf{5 7 . 7 5}$ & $\mathbf{0 . 9 9 8 5}$ \\
& CT & $\mathbf{5 9 . 3 0}$ & 0.9995 \\
& Ultrasound & $\mathbf{5 7 . 6 2}$ & 0.9990 \\
\hline & X-Ray & 55.06 & 0.9230 \\
Thanki, Borra et & MRI & 48.99 & 0.9692 \\
al. 2017 [15] & CT & 47.18 & 0.9827 \\
& Ultrasound & 50.27 & 0.9551 \\
\hline & X-Ray & 46.36 & NA \\
Parah, Ahad et al. & MRI & NA & NA \\
2017[16] & CT & NA & NA \\
& Ultrasound & NA & NA \\
\hline \multirow{2}{*}{ Gangadhar, } & X-Ray & NA & NA \\
Akula et al. 2018 & MRI & 48.53 & 0.9932 \\
{$[17]$} & CT & 43.25 & $\mathbf{1 . 0 0 0 0}$ \\
& Ultrasound & 45.01 & $\mathbf{1 . 0 0 0 0}$ \\
\hline \multirow{2}{*}{ Novamizanti, } & X-Ray & 53.54 & 0.9441 \\
Wahidah et al. & MRI & 54.24 & 0.6218 \\
2020 [19] & CT & 54.02 & 0.7968 \\
& Ultrasound & 55.39 & 0.6681 \\
\hline
\end{tabular}

above 0.9 is always present for all cases. This kind of cropping attack is serious and we believe that current watermarking schemes would not survive such an attack with the same NC values obtained in this work. The proposed scheme is specially designed to withstand various attacks that could affect different frequency values. Thence, only one of the watermarks extracted from the two frequency coefficients will be selected after each attack depending on $\mathrm{NC}$ values achieved.

In Table 2 we compared our scheme to the other works in the literature, the comparison is in terms of perceptual quality (PSNR in $\mathrm{dB}$ ) and robustness (NC value) under no attack. To compare the performance of the proposed method under various attacks, Table 4 illustrates the performance of our scheme against the same works listed in Table 2. It can be clearly seen from Table 2 and Table 4 that the proposed scheme outperformed the other works where the closest results obtained from [15] achieved an average PSNR value of $50.37 \mathrm{~dB}$ while the other competitive results are in [19] which achieved an

Table 3. Imperceptibility and robustness comparison with state-of-the-art work without attacks for other types of images

\begin{tabular}{|c|c|c|c|}
\hline Scheme & Image & PSNR & NC \\
\hline & Lena & $\mathbf{5 5 . 1 7}$ & 0.9949 \\
Propsed & Baboon & $\mathbf{5 4 . 8 5}$ & 0.9985 \\
& Peppers & $\mathbf{5 5 . 0 8}$ & 0.9990 \\
\hline Rakhmawati, & Lena & 41.66 & NA \\
Suwadi et al. & Baboon & 41.66 & NA \\
2020 [20] & Peppers & 41.26 & NA \\
\hline Setyono, & Lena & 45.22 & $\mathbf{1}$ \\
Setiadi et al. & Baboon & 45.60 & $\mathbf{1}$ \\
2020 [21] & Peppers & 45.47 & $\mathbf{1}$ \\
\hline Marjuni and & Lena & 43.42 & 0.9998 \\
Nurhayati 2021 & Baboon & 43.72 & 0.9999 \\
[22] & Peppers & 43.78 & 0.9997 \\
\hline
\end{tabular}




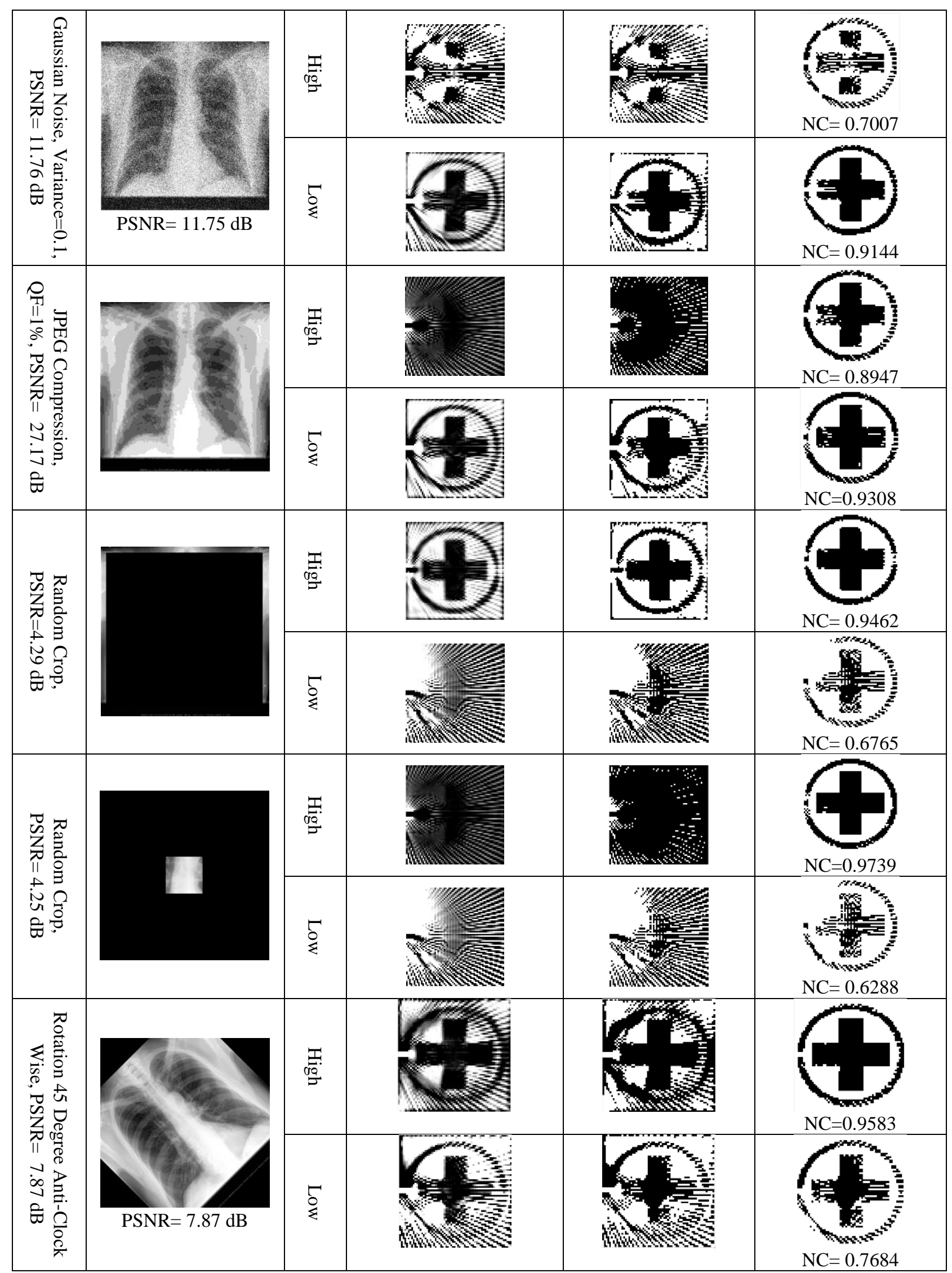

Figure. 7 Proposed scheme performance for $\mathrm{x}$-ray image against gaussian noise attack with $\mathrm{V}=0.1, \mathrm{JPEG}$ with QF=1 \%, random crop and rotation attacks 
Table 4. Robustness comparison with other works in terms of NC, for x-ray image

\begin{tabular}{|c|c|c|c|c|c|c|}
\hline Scheme & $\begin{array}{c}\text { JPEG, } \\
\mathrm{Q}=90 \%\end{array}$ & $\begin{array}{c}\text { JPEG, } \\
\mathrm{Q}=50 \%\end{array}$ & $\begin{array}{c}\text { Histogram } \\
\text { Equalization }\end{array}$ & $\begin{array}{c}\text { Median } \\
\text { Filtering } \\
2 \mathrm{X} 2\end{array}$ & $\begin{array}{c}\text { Gaussian } \\
\text { Nosie, } \\
\text { Var.=0.01 }\end{array}$ & $\begin{array}{c}\text { Salt and } \\
\text { Pepper } \\
\text { Nosie, } \\
\text { Den.=0.1 }\end{array}$ \\
\hline Proposed & $\mathbf{0 . 9 9 7 9}$ & $\mathbf{0 . 9 9 2 3}$ & $\mathbf{0 . 9 8 6 2}$ & $\mathbf{0 . 9 9 8 5}$ & $\mathbf{0 . 9 8 3 6}$ & $\mathbf{0 . 9 5 8 8}$ \\
\hline Thanki, Borra et al. 2017 [15] & 0.9713 & 0.7125 & 0.9689 & 0.9538 & 0.6377 & 0.7532 \\
\hline Parah, Ahad et al. 2017[16] & 0.5487 & NA & 0.5091 & NA & NA & 0.9501 \\
\hline Gangadhar, Akula et al. 2018 [17] & NA & NA & 0.9835 & NA & NA & NA \\
\hline Novamizanti, Wahidah et al. 2020 [19] & 0.9265 & NA & 0.8909 & 0.9570 & 0.9770 & 0.9473 \\
\hline
\end{tabular}

average PSNR value of $54.29 \mathrm{~dB}$. On the other hand, our proposed scheme achieved an average PSNR value of $57.61 \mathrm{~dB}$. Moreover, in terms of robustness the proposed scheme surpasses the works from Table 2 as illustrated in Table 4. Also, we compared our scheme to the state-of-the-art works in terms of PSNR and NC values under no attack as illustrated in Table 3.

\section{Conclusion}

This work presented a robust yet imperceptible watermarking scheme by securely embedding patient private information within medical images. In this context, hybrid Multiscale/Multiresolution frequency coefficients selection is employed using the Fast Discrete Curvelet Transform (FDCT) in conjunction with Singular Value Decomposition (SVD). In addition, the Radon Transform (RT) was applied on the watermarks before embedding to increase the robustness and security of the proposed scheme. Experimental results confirmed the efficiency of the proposed scheme with an average PSNR of $55 \mathrm{~dB}$ for images from the MedPix database. Various attacks were performed to evaluate the proposed method's robustness. Comparisons with related work confirmed the proposed scheme's efficiency in terms of perceptibility and imperceptibility.

\section{Conflict of interest}

The authors declare no conflict of interest.

\section{Author contributions}

A. A. Mohammed carried out the design of the watermarking scheme, performed the experiments, and drafted the manuscript. M. A. M. Abdullah guided the work, supervised the experimental design, and helped in drafting the manuscript. S. R. Awad participated in the comparison experiments and helped in drafting the manuscript. F. S. Alghareb participated in the design of the scheme and helped in drafting the manuscript. All authors read and approved the final manuscript."

\section{References}

[1] S. Haddad, G. Coatrieux, A. M. Gaudry, and M. Cozic, "Joint Watermarking-Encryption-JPEGLS for Medical Image Reliability Control in Encrypted and Compressed Domains", IEEE Transactions on Information Forensics and Security Vol. 15, pp. 2556-2569, 2020.

[2] D. N. Ramirez, M. C. Hernandez, M. N. Miyatake, and H. P. Meana, "Efficient Management of Ultrasound Images using Digital Watermarking", IEEE Latin America Transactions, Vol. 18, No. 08, pp. 1398-1406, 2020.

[3] P. Garg and R. R. Kishore, "Performance comparison of various watermarking techniques", Multimedia Tools and Applications, Vol. 79, No. 35, pp. 25921-25967, 2020.

[4] A. A. Mohammed, H. H. Maraş, and E. Elbasi, "A new robust binary image embedding algorithm in discrete wavelet domain", In: Proc. of IEEE 8th International Conf. on Application of Information and Communication Technologies (AICT), Astana, Kazakhstan, pp. 1-7, 2014.

[5] A. A. Mohammed, B. A. Jebur, and K. M. Younus, "Hybrid DCT-SVD Based Digital Watermarking Scheme with Chaotic Encryption for Medical Images", IOP Conference Series: Materials Science and Engineering, Mosul, Nineveh, Vol. 1152, No. 1, p. 012025, 2021.

[6] D. B. Taha, T. B. Taha, N. B. A. Dabagh, R. Ngadiran, and P. Ehkan, "Digital Image Watermarking Algorithm Based On Texture Masking Model", Journal of Engineering Science and Technology, Vol. 14, No. 6, pp. 3347-3360, 2019.

[7] J. Liu, J. Ma, J. Li, M. Huang, N. Sadiq, and Y. $\mathrm{Ai}$, "Robust Watermarking Algorithm for Medical Volume Data in Internet of Medical Things", IEEE Access, Vol. 8, pp. 93939-93961, 2020 . 
[8] F. N. Thakkar and V. K. Srivastava, "A blind medical image watermarking: DWT-SVD based robust and secure approach for telemedicine applications", Multimedia Tools and Applications, Vol. 76, No. 3, pp. 3669-3697, 2017.

[9] S. A. Parah, J. A. Sheikh, F. Ahad, N. A. Loan, and G. M. Bhat, "Information hiding in medical images: a robust medical image watermarking system for E-healthcare", Multimedia Tools and Applications, Vol. 76, No. 8, pp. 10599-10633, 2017.

[10] S. Nagpal, S. Bhushan, and M. Mahajan, "An enhanced digital image watermarking scheme for medical images using neural network, DWT and RSA", International Journal of Modern Education and Computer Science, Vol. 8, No. 4, pp. 46-56, 2016.

[11] P. Kishore, M. S. Rao, C. Prasad, and D. A. Kumar, "Medical image watermarking: run through review", ARPN Journal of Engineering and Applied Sciences, Vol. 11, No. 5, pp. 28822899, 2016.

[12] A. Mahmood, "Adaptive approaches for medical imaging security", 2015.

[13] A. K. Singh and M. Dave, "Hybrid technique for robust and imperceptible dual watermarking using error correcting codes for application in telemedicine", International Journal of Electronic Security and Digital Forensics, Vol. 6, No. 4, pp. 285-305, 2014.

[14] M. A. M. Abdullah, S. S. Dlay, and W. L. Woo, "Securing iris images with a robust watermarking algorithm based on discrete cosine transform", In: Proc. of International Conference on Computer Vision Theory and Applications, Berlin, Germany, Vol. 2, pp. 108114, 2015.

[15] R. Thanki, S. Borra, V. Dwivedi, and K. Borisagar, "An efficient medical image watermarking scheme based on FDCuT-DCT", Engineering Science and Technology, an International Journal, Vol. 20, No. 4, pp. 13661379, 2017.

[16] S. A. Parah, F. Ahad, J. A. Sheikh, and G. M. Bhat, "Hiding clinical information in medical images: A new high capacity and reversible data hiding technique", Journal of Biomedical Informatics, Vol. 66, pp. 214-230, 2017.

[17] Y. Gangadhar, V. S. G. Akula, and P. C. Reddy, "An evolutionary programming approach for securing medical images using watermarking scheme in invariant discrete wavelet transformation", Biomedical Signal Processing and Control, Vol. 43, pp. 31-40, 2018.
[18] A. A. Mohammmed, E. Elbasi, and O. M. Alsaydia, "An Adaptive Robust Semi-blind Watermarking in Transform Domain Using Canny Edge Detection Technique", In: Proc. of 44th International Conference on Telecommunications and Signal Processing (TSP), Brno, Czech Republic, pp. 10-14, 2021.

[19] L. Novamizanti, I. Wahidah, and N. P. D. P. Wardana, "A Robust Medical Images Watermarking Using FDCuT-DCT-SVD", International Journal of Intelligent Engineering and Systems, Vol. 13, No. 6, pp. 266-278, 2020.

[20] L. Rakhmawati, S. Suwadi, and W. Wirawan, "Blind robust and self-embedding fragile image watermarking for image authentication and copyright protection with recovery capability", International Journal of Intelligent Engineering and Systems, Vol. 13, No. 5, pp. 197-210, 2020.

[21] A. Setyono and D. R. I. M. Setiadi, "An Image Watermarking Method Using Discrete Tchebichef Transform and Singular Value Decomposition Based on Chaos Embedding", International Journal of Intelligent Engineering and Systems, Vol. 13, No. 2, pp. 140-150, 2020.

[22] A. Marjuni and O. D. Nurhayati, "Robustness Improvement Against a Non-Geometrical Attacks of Lifting Scheme-Based Image Watermarking through Singular Value and Schur Decompositions", International Journal of Intelligent Engineering and Systems, Vol. 14, No. 4, pp. 217-230, 2021.

[23] E. J. Candès and D. L. Donoho, "Ridgelets: A key to higher-dimensional intermittency?", Philosophical Transactions of the Royal Society of London. Series A: Mathematical, Physical and Engineering Sciences, Vol. 357, No. 1760, pp. 2495-2509, 1999.

[24] E. Candes, L. Demanet, D. Donoho, and L. Ying, "Fast discrete curvelet transforms", Multiscale Modeling and Simulation, Vol. 5, No. 3, pp. 861899, 2006.

[25] MedPix ${ }^{\mathrm{TM}}$ Medical Image Database available at https://medpix.nlm.nih.gov/home. 